\title{
Effect of Dietary Polyunsaturated Phospholipid on the Chemical Composition of Mesenteric Lymph Chylomicrons and the Excretion of Steroids into Bile and Feces in Rat
}

\author{
Katsumi IMAIzUmI, Màsakazu MURATA, and Michihiro SUGANo ${ }^{1}$ \\ Laboratory of Nutrition Chemistry, Kyushu University School \\ of Agriculture, Higashi-ku, Fukuoka 812, Japan
}

(Received August 17, 1981)

\begin{abstract}
Summary The effect of soybean phospholipid (SP) on the chemical composition of lymph chylomicrons and excretion of steroids into bile and feces was examined. Rats were meal-fed ( $9 \mathrm{am}-10 \mathrm{am})$ or ad libitum-fed on diets containing phospholipid and neutral lipid (soybean oil or corn oil) for 2 weeks in order to assess the amount of chylomicrons secreted from the intestine during the absorption or post-absorption of dietary lipids. In meal-fed rats, dietary SP decreased the concentration of surface components (phospholipid, cholesterol and apoprotein A-I) of large chylomicrons and increased the core components (triglyceride) of small chylomicrons. In ad libitum-fed rats given SP, the concentration of triglyceride in small chylomicrons appeared to increase. Dietary SP increased the relative proportion of linoleic acid and decreased arachidonic acid at the 2position of chylomicron-phospholipids. The calculated diameter of large and small chylomicron particles in meal-fed rats given SP increased and the particle number per ml lymph decreased compared to those values in rats given neutral lipid. The particle size of the small chylomicron in ad libitum-fed rats given SP also increased. Apoprotein A-I in lymph $d>1.006 \mathrm{~g} / \mathrm{ml}$ decreased in the SP-fed rats. The permeability of glucose-6phosphate to microsomal membrane increased in the SP-fed rats. The excretion of neutral, but not acidic steroids into the bile and feces was increased in SP-fed rats.

The results suggest that dietary SP inhibits the supply of the surface components of chylomicrons to the chylomicron precursors by interfering with absorption of the lipids in the upper part of the intestine and altering the subsequent metabolism of these lipids and apoproteins in the intestinal cells.
\end{abstract}

Key Words phospholipid, chylomicrons, particle size, bile steroids, fecal steroids

1 今泉勝己, 村田昌一, 菅野道廣

Abbreviations: SP, soybean phospholipid; CO, corn oil; SO, soybean oil; apo, apolipoprotein. 
Phospholipid is an integral component of all membranes and an important constituent of the diet. Large daily and individual variations may be expected in the dietary intake of humans depending on the type of diet. The average daily intake of phospholipid in man in our university refectory was almost $3 \mathrm{~g}$ (unpublished observation). This amount is almost comparable to the observation of Borgström (1). Phospholipid enters the intestinal lumen from the bile as well as from the diet. Bile secretion in man contributes about $12 \mathrm{~g}$ of phospholipid per day (2). Thus endogenous phospholipid production in man appears to be quantitatively more important than dietary phospholipid. However, this is not so in the case of rats given commercial rations. The daily intake, in rats weighing $200 \mathrm{~g}$, of the phospholipid from the commercial rat chow was about $200 \mathrm{mg}$ (unpublished observation) and daily secretion of bile phospholipid was estimated to be about $150 \mathrm{mg}(3)$. The phospholipid in bile is present in mixed micelles together with bile salts and cholesterol(4), whereas dietary phospholipid occurs mainly in cellular membranes and other lipoprotein structures. Therefore, this difference in the lumen may affect absorption and subsequent metabolism of nutrient. In this regard, recent papers by Mansbach (5) and Clark (6) have shown that luminal phosphatidylcholine infused intraduodenally with triglyceride at 4-5 times the biliary phosphatidylcholine secretion rate affects chylomicron lipid secretion plus phospholipid composition and synthesis in lymph fistula rats.

In the previous paper, we showed that soybean phospholipid (SP), given to rats at the level of $100 \mathrm{~g} / \mathrm{kg}$ diet, caused the alteration of the chemical composition of serum lipoproteins(7). In the present study we have examined the chemical composition of lymph chylomicrons secreted from the rat intestine during and after absorption of the dietary SP and neutral lipid.

\section{MATERIALS AND METHODS}

Experimental animals and diets. Male Wistar rats weighing 300-350 g housed in wire mesh cages under well-ventilated conditions with a $12 \mathrm{hr}$ light cycle (6 am-6 pm) were used. Soybean phospholipid (SP), soybean oil (SO) or corn oil (CO) was added to the semipurified diet at the level of $10 \%$ as described previously (7). In experiment 1, rats were fed ad libitum for 2 weeks (ad lib.-fed rats). In experiment 2, the diets were given to rats for $1 \mathrm{hr}$ from 9 am to 10 am every day for 2 weeks (meal-fed rats). Feces from ad lib.-fed rats were collected over $48 \mathrm{hr}$.

Mesenteric lymph and bile collection. Intestinal lymph duct cannulation was performed as described previously $(8)$, except that the duodenum was not cannulated. Silicone tubing $(0.5 \mathrm{~mm}$ ID $\times 1.0 \mathrm{~mm}$ OD) was inserted into the main mesenteric lymph duct. The bile duct was cannulated with polyethylene tubing (PE 10). The rats were put in the restraining cages (9) during the collection of the lymph or bile. The lymph was collected for approximately $5 \mathrm{hr}$, from $10 \mathrm{am}$ in ad lib.-fed rats and from 11 am in meal-fed rats, respectively. The bile was collected for $1 \mathrm{hr}$ from $11 \mathrm{am}$. The lymph and bile were collected in a tube placed in crushed ice. 
Preparation of lymph lipoprotein. Lymph lipoproteins were fractionated as described previously(8). Briefly, large chylomicrons were separated by centrifugation of the fresh lymph layered beneath $0.15 \mathrm{M} \mathrm{NaCl}$ containing $0.04 \%$ EDTA at $10^{\circ} \mathrm{C}$ in a 40.3 rotor for $1.6 \times 10^{6} \mathrm{~g}$-av min. The small chylomicrons were separated by centrifugation of the infranatant of large chylomicrons at $1 \times 10^{8} \mathrm{~g}$-av min. The infranatant of the small chylomicrons was designated as the fraction having a density greater than $1.006 \mathrm{~g} / \mathrm{ml}(d>1.006 \mathrm{~g} / \mathrm{ml})$. In one experiment, lymph lipoproteins were also separated by gel permeation chromatography on a $0.9 \mathrm{~cm} \times 92 \mathrm{~cm}$ column of $4 \%$ agarose (Sepharose 4B, Pharmacia Fine Chemicals Inc.) using an equilibrating and running buffer containing $0.2 \mathrm{M} \mathrm{NaCl}, 0.001 \mathrm{M}$ EDTA, $0.02 \% \mathrm{NaN}_{3}, \mathrm{pH} 7.2$. Samples were applied in a final volume of $2 \mathrm{ml}$ containing $20 \%$ sucrose (10).

Electroimmunoassay procedure for lymph apo $A$-I. The determination of the lymph apo A-I was performed according to the method of Laurell (11) as described previously(7). Since mesenteric lymph lipoprotein contained large and poorly diffusing chylomicrons, the electroimmunoassay procedure which was successful for the quantitation of the serum apo A-I was validated by comparison of apo A-I concentrations in native chylomicrons and chylomicrons delipidated by diisopropyl ether-butanol mixture (12). The apo A-I contents of the total protein in the native and delipidated chylomicrons were identical: $37.0 \pm 0.7 \%(n=3)$ for the native chylomicron or $37.3 \pm 1.0(n=3)$ for the delipidated chylomicron, respectively. These values were in good agreement with those obtained previously by radioimmunoassay $(31.2 \%)$ and column chromatography $(38.2 \%)(8)$.

Determination of the permiability of the microsomal membrane in the intestine. The permiability of the microsomal membrane of the intestine from ad lib.-fed rats was tested by measuring the activity of glucose-6-phosphatase, as described by Arion et al. (13). The intestine was cut into sections opened up logitudinally on an iced glass plate, and the mucosa carefully cleaned by delivering a jet of $0.9 \% \mathrm{NaCl}$ and light wiping tissue paper. The entire mucosa, down to the level of the crypts, was removed from the intestine by scraping with a glass slide. The mucosa was collected in $30 \mathrm{ml}$ of $0.278 \mathrm{M}$ mannitol containing $0.01 \mathrm{M}$ Tris, $\mathrm{pH} 7.4$, and homogenized in a Teflon-glass, motor-driven homogenizer. A microsomal preparation was obtained(14), and the glucose-6-phosphatase activity was studied as described by Nodlie (15).

Analytical methods. Lipids were extracted in chloroform-methanol mixture and triglyceride; phospholipid and cholesterol were determined as described previously(7). Separation of neutral lipid and phospholipid was carried out by thinlayer chromatography on Silica gel G (Merck) using a solvent mixture of petroleum ether-ether-acetic acid(16). Gas-liquid chromatographic analysis of fatty acid methylesters was performed as reported previously (17). Analyses of the bile and fecal neutral and acidic steroids were performed as described previously (18) according to the methods of Miettinen et al.(19) and Grundy et al.(20), respectively. $5 \alpha$-Cholestane and $5 \beta$-cholanoic acid were used as internal calibration 
standards.

Calculation. The particles of large or small chylomicrons were assumed to be spherical and entirely composed of apolar lipids in the core of radius $R_{2}$ and constant thickness of the polar surface shell 21.5 A(21). Particle densities were calculated from the partial specific volumes of the constituents; 1.093 for triglyceride; 1.044 for cholesterol ester; 0.968 for free cholesterol; 0.970 for phospholipid and 0.705 for protein $(21)$. The radius, $R_{1}$, of the chylomicron particle was calculated as described by Redgrave and Carlson (22). Since the total volume, $V_{1}$, of all constituents of a chylomicron fraction and the volume, $\mathrm{V}_{2}$ of the apolar constituents can be calculated per $\mathrm{ml}$ in the isolated fractions, the ratio $R_{2} / R_{1}=C=$ $\left(V_{2} / V_{1}\right)^{1 / 3}$ can be obtained. Therefore, it follows that $R_{1}-R_{2}=21.5 \mathrm{~A}$ and hence $R_{1}=21.5 /(1-C)$. Particle molecular weight in daltons was then calculated as the product of particle weight and Avogadro number. Particle numbers were calculated by dividing the value of a particle of diameter $2 R_{1}$ into the total chylomicron volume.

The data were statistically analyzed using the Students' $t$ test.

\section{RESULTS}

\section{Mesenteric apo A-I and lipids}

In order to determine the effect of soybean phospholipid on intestinal lipoprotein synthesis and secretion into the mesenteric lymph at the post absorptive and during the active absorptive state, lymph was obtained from both ad lib.- and meal-fed rats. Duodenum perfusion with a physiological solution in order to maintain preferable lymph flow was not used, since we wanted to determine the composition of mesenteric lymph produced in the native intestine in regularly fed rats. One of the disadvantages of the present operation was a low lymph flow (0.3$0.8 \mathrm{ml} / \mathrm{hr}$ as the average of several experiments) compared to the previous experiment where the duodenum was perfused with physiological saline $(1-1.5 \mathrm{ml} / \mathrm{hr})(8)$. Therefore, the present results were expressed as concentrations instead of secretion rates. Since lymph flow rates as shown in Table 1 were comparable between the SPand SO- or CO-fed rats, we believe that making a comparison between both dietary treatments is rational.

As shown in Table 1, the concentration of mesenteric lymph apo A-I in SP-fed rats was significantly lower than that in rats fed on the $\mathrm{CO}$ diet ad lib. or than that of SO meal-feeding rats. On ad lib. feeding, the concentration of apo A-I in SP-fed rats was significantly lower only in the $d>1.006 \mathrm{~g} / \mathrm{ml}$ fraction than that of CO-fed rats. On meal feeding, the significant reduction of apo A-I in SP-fed rats was observed in large chylomicrons and the $d>1.006 \mathrm{~g} / \mathrm{ml}$ fraction. In the $d>1.006 \mathrm{~g} / \mathrm{ml}$ fraction, apo A-I was recovered at the 59-62\% level in ad lib.-fed rats and the $38-42 \%$ level in meal-fed rats. Since apo A-I in the $d>1.006 \mathrm{~g} / \mathrm{ml}$ fraction in mesenteric lymph might be artifacts dissociated during the ultracentrifugation from the chylomicrons (10), lymph was subjected to gel permeation chromatography as shown in Fig. 


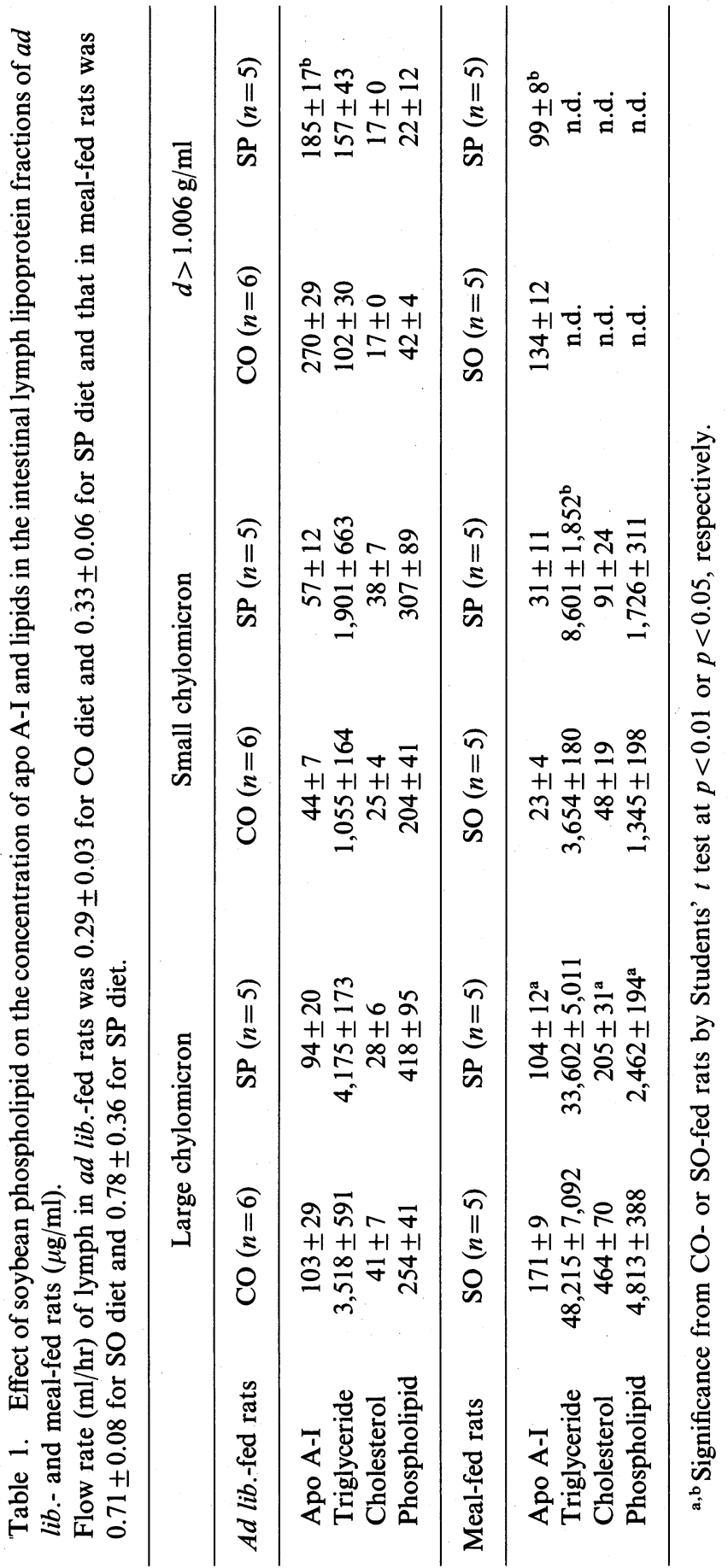

Vol. 28, No. 3, 1982 


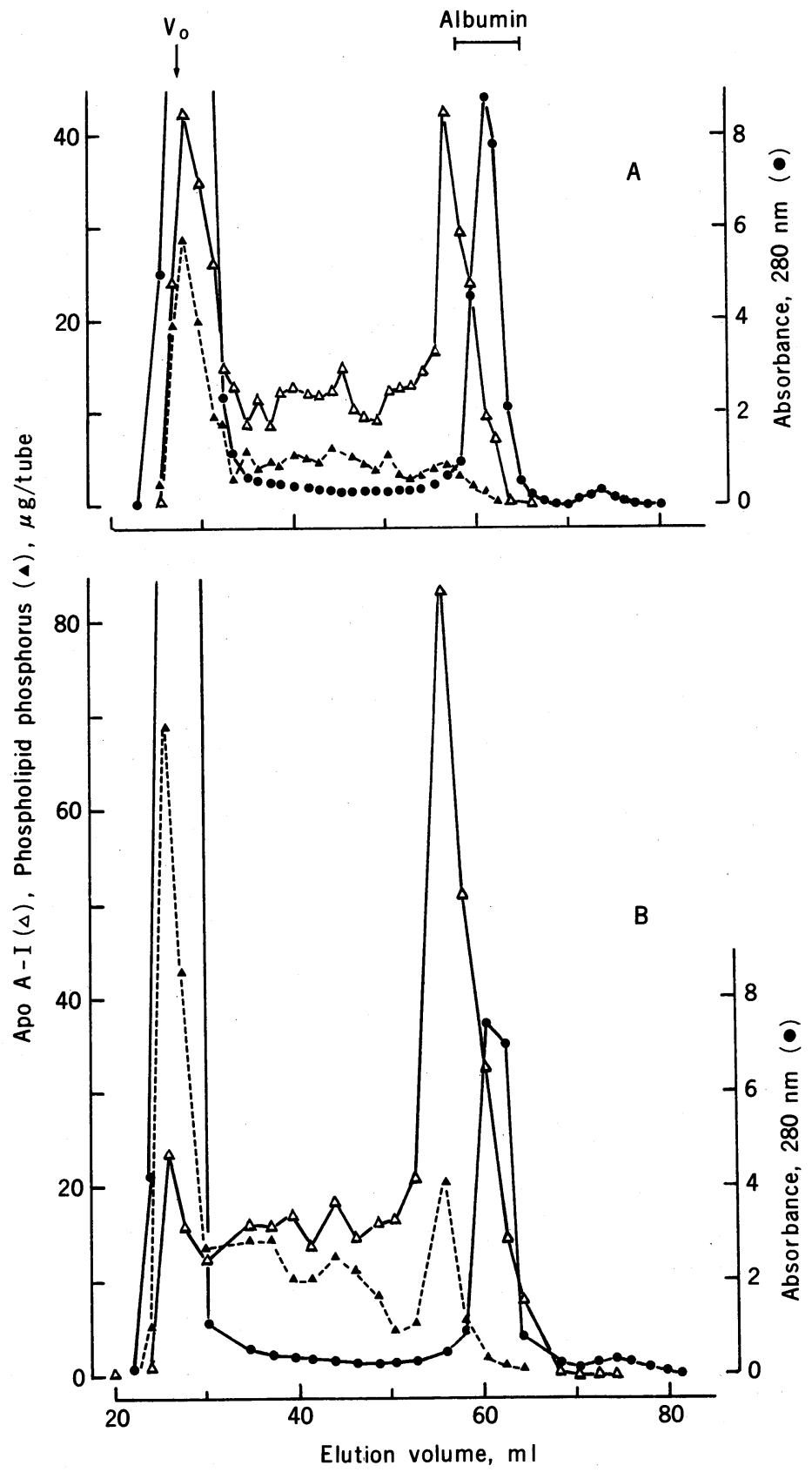

Fig. 1. Chromatography of $1.5 \mathrm{ml}$ lymph on $0.9 \times 92 \mathrm{~cm}$ column of Sepharose 4B. A: lymph from meal-fed rat, B: lymph from ad lib.-fed rat. 
1. Large chylomicrons were eluted in the void volume, and small chylomicrons from the void volume to the elution volume, a region of approximately $50 \mathrm{ml}$. Two prominent peaks of apo A-I, eluting in the void volume and in the 50-60 ml regions were observed. The recovery of apo A-I in the combined eluate of $50-65 \mathrm{ml}$ fractions was $31.7 \%$ in meal-fed rats and $53.9 \%$ in ad lib.-fed rats, respectively. The phospholipid elution pattern was similar to that of the apo A-I. Therefore, the dissociation of apo A-I from chylomicrons during ultracentrifugation is relatively small, if any, under the present ultracentrifugation conditions.

Table 1 also shows the distribution of lipids in the mesenteric lymph lipoprotein fractions of ad lib.- and meal-fed rats. In ad lib.-fed rats, less than $10 \%$ of triglyceride and phospholipid, but approximately $20 \%$ of lymph cholesterols were found in the $d>1.006 \mathrm{~g} / \mathrm{ml}$ fraction. In ad lib.-fed rats, the concentration of lipids was not significantly different between the rats fed on SP and CO even though triglyceride and phospholipid appeared to be higher in SP-fed rats. In meal-fed rats, triglyceride, phospholipid and cholesterol in large chylomicrons were all lower in SP- than in SO-fed rats. In contrast, the concentrations of lipids in small chylomicrons were higher in SP- than in SO-fed rats.

From the chemical compositions of large and small chylomicrons, average molecular parameters of large and small chylomicrons were estimated by the calculation as described in MATERIALS AND METHODS (Table 2). Alterations in the physicochemical parameters of the chylomicron were observed in the meal-fed rats to be more remarkable than those in the ad lib.-fed rats. In meal-fed rats, dietary SP increased the diameter (1.4- and 1.7-fold) and the molecular weight (2.4- and 4.6fold) of the large and small chylomicrons. On the other hand, the particle numbers per $\mathrm{ml} \mathrm{lymph}$ of the large and small chylomicrons in SP-fed rats decreased by $28 \%$ and $44 \%$, respectively, of those in SO-fed rats. In ad lib.-fed rats given dietary SP,

Table 2. Effect of soybean phospholipid on the molecular parameters of large and small chylomicrons.

\begin{tabular}{crcc}
\hline & $\begin{array}{c}\text { Diameter } \\
(\AA)\end{array}$ & $\begin{array}{c}\text { Molecular weight } \\
\left(\text { daltons } \times 10^{-6}\right)\end{array}$ & $\begin{array}{c}\text { Number of particles } \\
\left(\times 10^{-12} / \mathrm{ml} \text { lymph }\right)\end{array}$ \\
\hline Ad lib.-fed rats & & & \\
CO Large chylomicron & 1,048 & 357 & 7.1 \\
$\quad$ Small chylomicron & 606 & 68 & 12.6 \\
SP Large chylomicron & 1,024 & 322 & 12.3 \\
$\quad$ Small chylomicron & 729 & 117 & \\
\hline Meal-fed rats & & & 41.8 \\
SO Large chylomicron & 1,387 & 785 & 11.6 \\
Small chylomicron & 467 & 31 & 45.0 \\
SP Large chylomicron & 1,870 & 1,918 & \\
$\quad$ Small chylomicron & 782 & 142 & \\
\hline
\end{tabular}

Vol. 28, No. 3, 1982 
Table 3. Effect of soybean phospholipid on the fatty acids composition of triglyceride and phospholipid in large and small chylomicrons in ad lib.-fed rats (weight \%). ${ }^{a}$

\begin{tabular}{lccccccc}
\hline & $16: 0$ & $16: 1$ & $18: 0$ & $18: 1$ & $18: 2$ & $18: 3$ & $20: 4$ \\
\hline $\begin{array}{l}\text { Triglyceride } \\
\quad \text { Large chylomicron }\end{array}$ & & & & & & & \\
$\quad$ CO & 15.8 & 1.6 & 5.3 & 25.5 & 45.3 & $\operatorname{tr}$ & 6.5 \\
$\quad$ SP & 19.3 & 1.9 & 4.9 & 14.3 & 48.4 & 5.9 & 3.4 \\
$\quad$ Small chylomicron & & & & & & & \\
$\quad$ CO & 19.0 & 2.0 & 7.7 & 23.6 & 40.4 & $\operatorname{tr}$ & 7.3 \\
SP & 23.4 & 2.3 & 8.0 & 16.9 & 43.7 & 2.6 & 2.1 \\
\hline Phospholipid & & & & & & & \\
$\quad$ Large chylomicron & & & & & & & \\
$\quad$ CO & 22.2 & 2.9 & 18.8 & 8.1 & 29.7 & $\operatorname{tr}$ & 18.3 \\
$\quad$ SP & 23.7 & 1.8 & 20.9 & 6.6 & 39.9 & $\operatorname{tr}$ & 7.3 \\
Small chylomicron & & & & & & & \\
$\quad$ CO & 26.5 & 3.9 & 23.6 & 11.6 & 27.6 & $\operatorname{tr}$ & 7.0 \\
SP & 27.0 & 2.1 & 27.1 & 9.1 & 32.1 & $\operatorname{tr}$ & 2.6 \\
\hline
\end{tabular}

${ }^{\text {a }}$ Pooled samples were analyzed.

Table 4. Effect of soybean phospholipid on the fatty acid composition of triglyceride and phospholipid in large and small chylomicrons in meal-fed rats (weight \%).

\begin{tabular}{|c|c|c|c|c|c|c|c|}
\hline & $16: 0$ & $16: 1$ & $18: 0$ & $18: 1$ & $18: 2$ & $18: 3$ & $20: 4$ \\
\hline \multicolumn{8}{|l|}{ Triglyceride } \\
\hline \multicolumn{8}{|c|}{ Large chylomicron } \\
\hline SO $(n=5)$ & $9.8 \pm 0.5$ & $1.0 \pm 0.2$ & $2.9 \pm 0.2$ & $18.3 \pm 1.9$ & $56.6 \pm 1.8$ & $9.5 \pm 0.5$ & $1.7 \pm 0.2$ \\
\hline $\mathrm{SP}(n=5)$ & $16.4 \pm 0.9^{\mathrm{a}}$ & $2.5 \pm 0.2^{\mathrm{a}}$ & $4.8 \pm 0.6^{c}$ & $15.5 \pm 1.4$ & $51.9 \pm 2.2$ & $6.6 \pm 0.6$ & $2.2 \pm 0.5$ \\
\hline \multicolumn{8}{|c|}{ Small chylomicron } \\
\hline SO $(n=5)$ & $14.8 \pm 0.8$ & $1.4 \pm 0.1$ & $4.8 \pm 0.2$ & $21.5 \pm 0.4$ & $47.3 \pm 0.9$ & $6.4 \pm 0.3$ & $3.7 \pm 1.4$ \\
\hline $\mathrm{SP}(n=5)$ & $17.5 \pm 0.6^{\mathrm{d}}$ & $2.7 \pm 0.2$ & $4.8 \pm 0.7$ & $15.1 \pm 0.6^{\mathrm{a}}$ & a $51.8 \pm 1.4$ & $6.2 \pm 0.7$ & $2.0 \pm 0.6$ \\
\hline \multicolumn{8}{|l|}{ Phospholipid } \\
\hline \multicolumn{8}{|c|}{ Large chylomicron } \\
\hline $\mathrm{SO}(n=5)$ & $17.7 \pm 0.3$ & $1.1 \pm 0.2$ & $16.4 \pm 0.4$ & $9.0 \pm 0.6$ & $38.9 \pm 1.0$ & $1.2 \pm 0.1$ & $15.4 \pm 1.3$ \\
\hline $\mathrm{SP}(n=5)$ & $18.9 \pm 0.4$ & $1.8 \pm 0.1$ & $15.2 \pm 0.6$ & $8.0 \pm 0.5$ & $46.7 \pm 1.8^{b}$ & $1.7 \pm 0.4$ & $7.8 \pm 1.4^{b}$ \\
\hline \multicolumn{8}{|c|}{ Small chylomicron } \\
\hline SO $(n=5)$ & $19.7 \pm 0.6$ & $2.4 \pm 0.2$ & $17.4 \pm 0.5$ & $11.6 \pm 1.2$ & $35.5 \pm 0.8$ & $1.1 \pm 0.1$ & $12.1 \pm 1.4$ \\
\hline $\mathrm{SP}(n=5)$ & $19.1 \pm 0.9$ & $2.1 \pm 0.2$ & $16.7 \pm 0.2$ & $9.0 \pm 0.4$ & $44.3 \pm 1.5^{\mathrm{d}}$ & $1.0 \pm 0.1$ & $7.8 \pm 1.5$ \\
\hline
\end{tabular}

a,b,c,d Significance from SO-fed rats by Students' $t$ test at $p<0.001, p<0.01, p<0.02$ and $p<0.05$, respectively. 


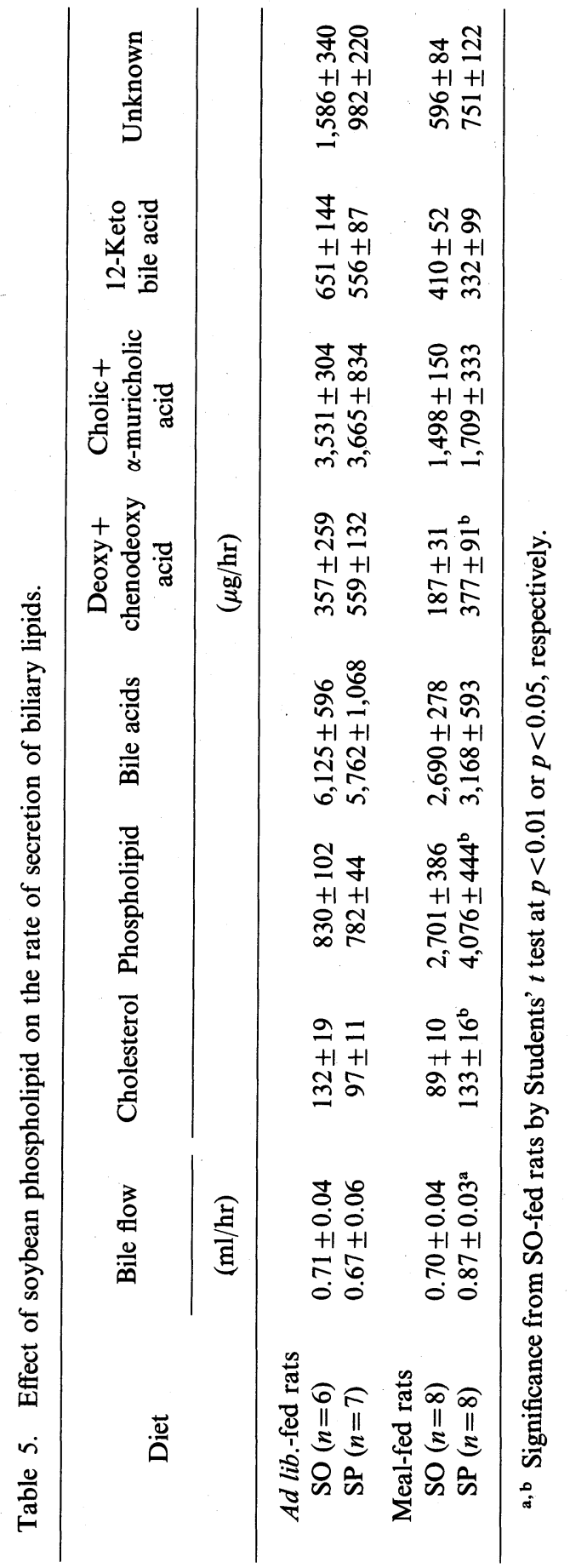

Vol. 28, No. 3, 1982 
the increase in the diameter (1.2-fold) and molecular weight (1.7-fold) was also observed in the small chylomicron. The particle numbers per ml lymph was almost the same in rats fed ad lib. with CO and SP.

The fatty acid compositions of triglyceride and phospholipid of large and small chylomicrons in ad lib.- and meal-fed rats are shown in Tables 3 and 4, respectively. The dietary SP given to ad lib.- and meal-fed rats appeared to increase in linoleic acid at the expense of arachidonic acid in the phospholipid. There was no change in the relative distribution of saturated fatty acids (palmitic and steric acids). The relative increase in the palmitic and linoleic acids at the expense of oleic and arachidonic acids (except for large chylomicrons in meal-fed rats) in the chylomicron triglyceride was observed in SP-fed rats. The calculated mass ratios of arachidonic to linoleic acid in the chylomicron was lower in the rats fed SP (data not shown). This was attributed to the remarkable reduction of the mass of the arachidonic acids in the lymph phospholipid in the SP-fed rats.

\section{Secretion of bile lipids}

Table 5 shows the rates of secretion of the biliary lipids of ad lib.- and meal-fed rats. In ad lib.-fed rats, there was no significant change in the secretion of the bile lipids between the rats fed SO and SP. In the meal-fed rats, however, cholesterol, phospholipid and one of the bile acids (deoxy and chenodeoxy cholic acid) increased. The relative distribution of phospholipid and bile acids in the bile lipids was remarkably different between the meal-fed and ad lib.-fed rats. The reason for this difference is not clear. The fatty acid compositions of the biliary phospholipid were almost identical in the rats fed SO and SP (Table 6).

\section{Fecal steroid excretion}

As shown in Table 7, dietary SP significantly increased the excretion of the fecal cholesterol and coprostanol in ad lib.-fed rats. Dietary SP also remarkably increased unidentified neutral steroids. The excretion of fecal bile acids, however, was not affected by the feeding of SP (Table 7).

Table 6. Effect of soybean phospholipid on the fatty acid composition of biliary phospholipid (weight \%).

\begin{tabular}{cccccccc}
\hline Diet & $16: 0$ & $16: 1$ & $18: 0$ & $18: 1$ & $18: 2$ & $18: 3$ & $20: 4$ \\
\hline Ad lib.-fed rats & & & & & & & \\
SO (n=6) & $31.6 \pm 1.7$ & $3.2 \pm 0.3$ & $5.9 \pm 0.2$ & $6.6 \pm 0.1$ & $34.5 \pm 1.2$ & $0.6 \pm 0.1$ & $15.4 \pm 0.8$ \\
SP $(n=7)$ & $31.6 \pm 1.5$ & $3.9 \pm 0.3$ & $5.0 \pm 0.4$ & $7.9 \pm 0.9$ & $34.3 \pm 0.4$ & $0.4 \pm 0.1$ & $15.4 \pm 2.8$ \\
Meal-fed rats & & & & & & & \\
SO (n=3) & $32.3 \pm 1.7$ & $3.2 \pm 0.3$ & $6.1 \pm 0.2$ & $6.7 \pm 0.1$ & $35.3 \pm 1.3$ & $0.6 \pm 0.1$ & $15.8 \pm 0.9$ \\
SP $(n=3)$ & $32.1 \pm 1.2$ & $3.9 \pm 0.3$ & $5.1 \pm 0.4$ & $8.0 \pm 1.0$ & $34.9 \pm 0.4$ & $0.4 \pm 0.1$ & $15.6 \pm 2.8$ \\
\hline
\end{tabular}




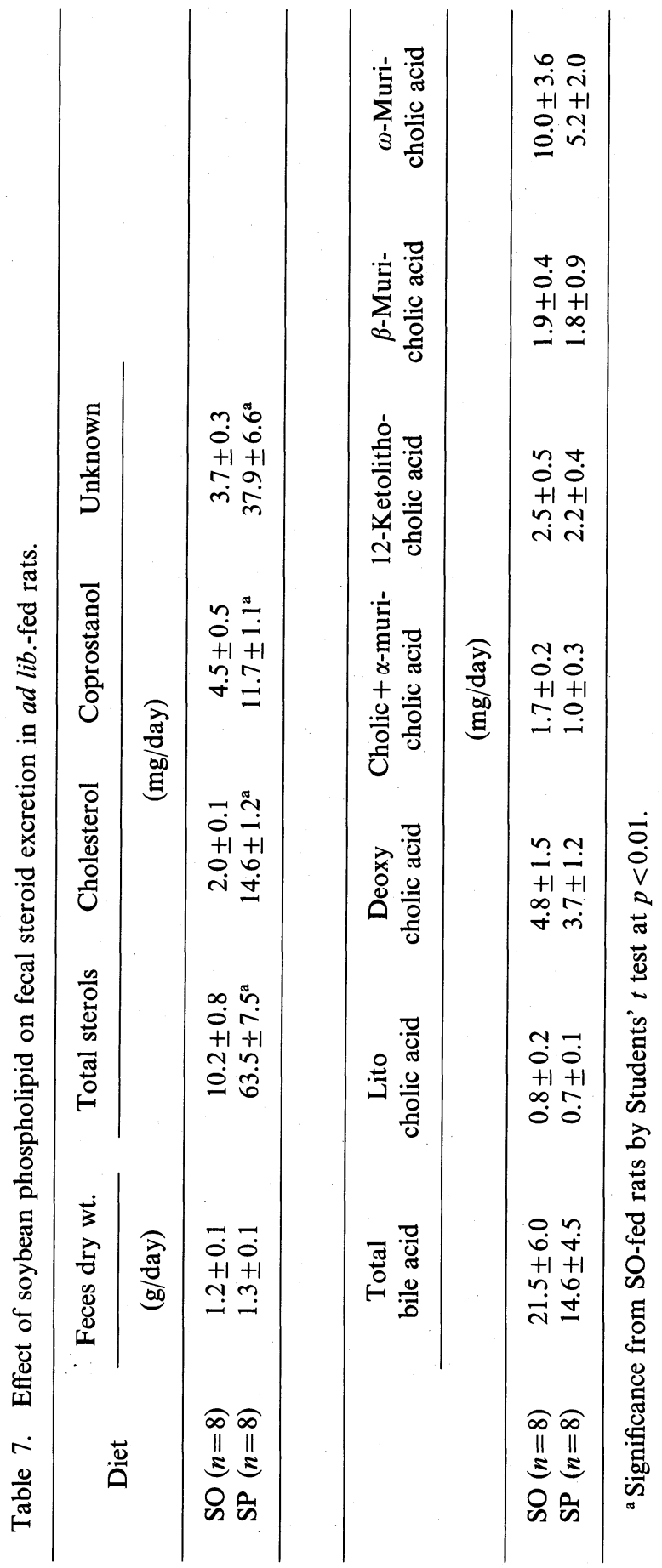

Vol. 28, No. 3, 1982 


\section{Glucose-6-phosphatase activity in the microsomes of intestinal mucosa}

Since the available data have suggested that glucose-6-phosphatase located in the endoplasmic reticulum or microsome in the liver and intestinal mucosa requires the transmicrosomal passage of its substrate before hydrolysis can occur (13), the permeability of the microsomes in the intestine to glucose-6-phosphate was examined. Glucose-6-phosphatase activity increased significantly in the rats fed SP compared to the rats fed SO $(51.9 \pm 3.9$ and $74.0 \pm 9.0 \mathrm{nmol} / \mathrm{mg}$ protein $\cdot \min$ for SOand SP-fed rats, respectively, $p<0.05$ ).

\section{DISCUSSION}

This study was carried out in order to clarify how dietary SP affects the synthesis and secretion of chylomicrons in the intestine of rats. The particle size of the chylomicron is distributed over a wide range $(400 \AA-6,000 \AA)$ and depends on the amount of the dietary triglyceride absorbed in the intestine (23). Larger particles (large chylomicrons) are secreted into lymph under active absorption, smaller particles (small chylomicrons) being secreted in the fasting state. In the present study, mesenteric lymph was collected from meal-fed and ad lib.-fed rats. Since the concentrations of the mesenteric lymph chylomicrons obtained from the meal-fed rats given SP and SO were seven- and eleven-fold higher, respectively, compared to those with the ad lib.-fed rats, the chylomicron triglyceride in the meal-fed rats is mainly derived from dietary lipids. In meal-fed rats, the concentration of the triglyceride in the chylomicrons obtained from SP-fed rats was about $80 \%$ of those from the SO-fed rats. This means that a considerable amount of the fatty acids in the phospholipid is utilized for chylomicron-triglyceride because the dietary SP used in this experiment contained approximately $35 \%$ neutral lipids. The utilization of the fatty acids in the phosphatidylcholine for the formation of the chylomicron triglyceride has been shown by radiolabeling the fatty acids in phosphatidylcholine (24). The contribution of the glycerophospholipids other than phosphatidylcholine to the formation of the chylomicron triglyceride remains to be established. Since phosphatidylcholine accounted for only $35 \%$ of phospholipids of SP (7), it is assumed that the fatty acids in glycerophospholipids other than phosphatidylcholine were also utilized for chylomicron triglyceride.

The present study demonstrated that dietary SP increased the particle size of the large and small chylomicrons obtained from the meal-fed rats and decreased the number of particles secreted into the lymph. This means that the dietary SP has an effect of expanding the core of the chylomicron particle composed of triglyceride in contrast to the surrounding surface composed of phospholipid, cholesterol and protein. The increase in the particle size of the chylomicron due to SP feeding was observed not only in the absorptive state (meal feeding) but also in the postabsorptive state (ad lib. feeding). The human experiment performed by Beil et al. (25) has shown the increase in small chylomicrons in plasma due to the feeding of soybean lecithin. In contrast to the care with human experiment, these workers have 
shown that rats infused continuously with lecithin through the duodenum secreted large chylomicrons into the mesenteric lymph to the same extent as did rats infused with safflower oil. The difference in the rat experiments performed by ourselves and Beil et al. might depend on experimental design.

The reason for the alteration of the physicochemical nature of the lymph chylomicron is not clear, but the following possible mechanisms can be proposed. First, dietary SP may increase the distal absorption of fatty acids from the dietary SP unabsorbed in the upper part of the intestine. Several workers have shown that large amounts of lecithin perfused into the lumen of the duodenum inhibits the absorption of long chain fatty acids and cholesterol in the proximal intestine $(26,27)$, and that the unhydrolyzed lecithin is absorbed in the distal intestine (28). Chylomicrons produced in the distal intestine are relatively rich in triglyceride and poor in surface components compared to those in the proximal intestine $(29,30)$.

As a second possible reason, dietary SP may increase the supply of the triglyceride and decrease the supply of the surface components for chylomicron synthesis. Luminal perfusion in vivo of lecthin increases the permeability of the intestinal microsomal membrane and hence decreases phosphocholinetransferase activity (31). It is well known that competition for the common substrate diglyceride between phosphocholinetransferase and diglyceride acyltransferase determines de novo synthesis of phosphatidylcholine and triglyceride (32). In addition, dietary SP appeares to decrease the supply of apo A-I, as shown in Table 1, one of the major protein components of chylomicrons (8). The apo A-I in the large chylomicron mainly derives from intestinal synthesis (33). It remains to be determined whether dietary SP decreases the supply of phosphatidylcholine and free cholesterol derived from bile and/or diet for chylomicron synthesis.

The fatty acid composition of the chylomicron phospholipid was measured in order to determine whether the 1-acyllysophosphatidylcholine in the intestinal lumen could be incorporated intactly into the chylomicron phospholipid. The phosphatidylcholine used in the SP diet contained $53 \%$ of linoleic acid at the 1-position(7). Lekim showed that linoleic acid at the 1-position, but not the 2-position of the oral phosphatidylcholine was incorporated intactly into the chylomicron phosphatidylcholine when doubly labeled dilinoleoylphosphatidylcholine with $\mathrm{H}^{3}$ and $\mathrm{C}^{14}$ was used (24). As shown in Tables 3 and 4, the relative proportion of the linoleic acid in the chylomicron phosphatidylcholine increased at the expense of arachidonic acid, and there was no reduction of the saturated fatty acids. Therefore, the direct incorporation of the dietary 1-linoleoyl phosphatidylcholine into the counterpart of the chylomicron will be quite small, if any.

The present results have shown that dietary SP increased the excretion of neutral steroid, but not bile acid, into the feces. In humans, similar results have been obtained by Greten et al.(34). Purified soybean phosphatidylcholine and egg yolk phospholipid mainly composed of phosphatidylcholine $(65 \%)$ and phosphatidylethanolamine $(35 \%)$ also increased fecal sterol excretion in rats (unpublished 
observation). A portion of the fecal neutral sterols would be attributed to the unabsorbed bile cholesterol since the previous in vivo and in vitro studies have shown that dietary phospholipid inhibits the intestinal absorption of cholesterol $(6,25-27)$. However, most of the neutral steroids in the feces should have a source other than bile, because daily excretion of bile cholesterol is about $3 \mathrm{mg}$ while that into the feces was $10 \mathrm{mg}$ in SO-fed rats, and $63 \mathrm{mg}$ in SP-fed rats, as shown in Table 7. The most probable source of the fecal sterols appears to be intestinal desquamated cells (35). Plant sterols contained in the dietary SP also contributed to the unidentified fecal sterols in rats given SP since the highest estimation of the daily intake of the plant sterols in the SP diet was about $10 \mathrm{mg}$. The contribution of the plant sterol to the alteration of lymph chylomicron composition, however, should be less important compared to that of the phospholipid itself because the neutral lipid fraction containing the phytosterols did not affect the pattern of metabolism of serum lipoprotein (7). It is not clear at present if the desquamation of the intestinal cells due to SP feeding accompanies increased cell renewal.

In the accompanying paper (7) we have shown that dietary SP decreases the concentration of serum cholesterol and apo A-I. The increased secretion of bile cholesterol, and the decreased amount of cholesterol and apo A-I in chylomicrons in SP-fed rats seemed to be responsible for the alteration of the serum lipoprotein patterns. In particular, decreased supply of the lymph apo A-I in the $d>1.006 \mathrm{~g} / \mathrm{ml}$ fraction, which is assumed to contain lymph high density lipoprotein, will bring about the reduction of the serum counterpart. Furthermore, physicochemical alteration of the lymph chylomicron due to dietary SP may also affect the metabolism of serum lipoprotein since an increase in the particle size of chylomicrons resulted in an increased clearance thereof in the serum (36).

Since the daily food of humans contains different kinds of phospholipids, future work should clarify the extent of the absorption of respective phospholipids and their effect on intestinal cell functions.

This study was supported in part by a Grant-in-Aid for Encouragement of Young Scientists (No. 476062) from the Ministry of Education, Science and Culture of Japan and by a research grant from the Foundation of Mishima Kaiun. The skillful technical assistance of T. Magarikaji is appreciated.

\section{REFERENCES}

1) Borgström, B. (1976): Phospholipid absorption, in Lipid Absorption: Biochemical and Clinical Aspect, ed. by Rommel, K., and Goebell, H., University Park Press, Baltimore, pp. 65-70.

2) Northfield, T. C., and Hofmann, A. F. (1975): Biliary output during three meals and an overnight fast. Gut, 16, 1-6.

3) Uchida, K., Nomura, Y., and Takeuchi, N. (1980): Effects of cholic acid, chenodeoxycholic acid, and their related bile acids on cholesterol, phospholipid and bile acid levels in serum, liver, bile and feces of rats. J. Biochem., 87, 187-194. 
4) Small, D. M., Bourgés, M. C., and Dervichian, D. G. (1966): Ternary and quarternary aqueous system containing bile salt, lecithin and cholesterol. Nature (Lond), 211, 816-818.

5) Mansbach, C. M., II. (1977): The origin of chylomicron phosphatidylcholine in the rat. J. Clin. Invest., 60, 411-420.

6) Clark, S. B. (1978): Chylomicron composition during duodenal triglyceride and lecithin infusion. Am. J. Physiol., 235, E183-E190.

7) Imaizumi, K., Murata, M., and Sugano, M. (1982): Effect of the dietary polyunsaturated phospholipid on the chemical composition of serum lipoproteins in rats. J. Nutr. Sci. Vitaminol., 28, 281-294.

8) Imaizumi, K., Fainaru, M., and Havel, R. J. (1978): Composition of proteins of mesenteric lymph chylomicrons in the rat and alterations produced upon exposure of chylomicrons to blood serum and serum lipoproteins. J. Lipid Res., 19, 712-722.

9) Girardet, R. E. (1974): A simple and inexpensive restraining cage for rats. J. Surg. Res., 17, 131-133.

10) Fainaru, M., Havel, R. J., and Imaizumi, K. (1977): Apoprotein content of plasma lipoproteins of the rat separated by gel chromatography or ultracentrifugation. Biochem. Med., 17, 347-355.

11) Laurell, C. B. (1966): Quantitative estimation of proteins by electrophoresis in agarose gel containing antibodies. Anal. Biochem., 15, 45-52.

12) Cham, B. E., and Knowles, B. R. (1976): A solvent system for delipidation of plasma or serum without protein precipitation. J. Lipid Res., 17, 176-181.

13) Arion, W. J., Ballas, L. M., Lange, A. J., and Wallin, B. K. (1976): Microsomal membrane permeability and the hepatic glucose-6-phosphatase system. Interactions of the system with D-mannose 6-phosphatase system. J. Biol. Chem., 251, 4901-4907.

14) Mansbach, C. M., II. (1973): Complex lipid synthesis in hamster intestine. Biochim. Biophys. Acta, 296, 386-400.

15) Nordlie, R. C., and Arion, W. J. (1966): Glucose-6-phosphatase. Methods Enzymol., 9, 619-625.

16) Kates, M. (1972): Techniques of Lipidology, in Laboratory Techniques in Biochemistry and Molecular Biology, ed. by Work, T. S., and Work, E., North-Holland Publishing Company, Amsterdam.

17) Sugano, M., Imaizumi, K., Cho, S., and Hori, K. (1969): Hepatotoxicity and lipid metabolism-I. Structure of liver triglyceride in rats dosed with carbon tetrachloride. Biochem. Pharmacol., 18, 1961-1970.

18) Sugano, M., Kamo, F., Ikeda, I., and Morioka, H. (1976): Lipid-lowering activity of phytostanols in rats. Atherosclerosis, 24, 301-309.

19) Miettinen, T. A., Ahrens, E. H., Jr., and Grundy; S. M. (1965): Quantitative isolation and gas-liquid chromatographic analysis of total dietary and fecal neutral steroids. $J$. Lipid Res., 6, 411-424.

20) Grundy, S. M., Ahrens, E. J. Jr., and Miettinen, T. A. (1965): Quantitative isolation and gas-liquid chromatographic analysis of total bile acid. J. Lipid Res., 6, 397-410.

21) Sata, T., Havel, R. J., and Jones, A. L. (1972): Characterization of subfractions of triglyceride-rich lipoproteins separated by gel chromatography from blood plasma of normolipemic and hyperlipemic humans. J. Lipid Res., 13, 757-768.

22) Redgrave, T. G., and Carlson, L. A. (1979): Changes in plasma very low density and low density lipoprotein content, composition, and size after a fatty meal in mormo- and hypertriglyceridemic man. J. Lipid Res., 20, 217-229.

23) Fraser, R. (1970): Size and lipid composition of chylomicrons of different Svedberg

Vol. 28, No. 3, 1982 
units of flotation. J. Lipid Res., 11, 60-65.

24) Lekim, D. (1976): On the pharmacokinetics of orally applied essential phospholipids (EPL), in Phosphatidylcholine; Biochemical and Clinical Aspects of Essential Phospholipids, ed. by H. Peeters. Springer-Verlag, Berlin, Heidelberg, New York, pp. 48-65.

25) Beil, F. U., and Grundy, S. M. (1980): Studies on plasma lipoproteins during absorption of exogenous lecithin in man. J. Lipid Res., 21, 525-536.

26) Rampone, A. J., and Long, L. R. (1977): The effect of phosphatidylcholine and lysophosphatidylcholine on the absorption and mucosal metabolism of oleic acid and cholesterol in vitro. Biochim. Biophys. Acta, 486, 500-510.

27) Hollander, D., and Morgan, D. (1980): Effect of plant sterols, fatty acids and lecithin on cholesterol absorption in vivo in the rat. Lipids, 15, 395-400.

28) Arnesjö, B., Nilson, A., Barrowman, J., and Borgström, B. (1969): Intestinal digestion and absorption of cholesterol and lecithin in the human. Scand. J. Gastroenterol., 4, 653-665.

29) Sabesin, S. M., Holt, P. R., and Clark, S. B. (1975): Intestinal lipid absorption; Evidence for an intrinsic defect of chylomicron secretion by normal rat distal intestine. Lipids, 12, 840-846.

30) Wu, A-L., Clark, S. B., and Holt, P. R. (1980): Composition of lymph chylomicrons from proximal or distal rat small intestine. Am. J. Clin. Nutr., 33, 582-589.

31) Mansbach, C. M., II., and Parthasarathy, S. (1979): Regulation of de novo phosphatidylcholine synthesis in rat intestine. J. Biol. chem., 254, 9688-9694.

32) Kennedy, E. P., and Weiss, S. B. (1956): The function of cytidine coenzyme in the biosynthesis of phospholipids. J. Biol. Chem., 222, 193-214.

33) Imaizumi, K., Havel, R. J., Fainaru, M., and Vigne, J-L. (1978): Origin and transport of the A-I and arginine-rich apolipoproteins in mesenteric lymph of rats. J. Lipid Res., 19, 1038-1046.

34) Greten, H., Raetzen, H., Stiehl, A., and Schettler, G. (1980): The effect of polyunsaturated phosphatidylcholine on plasma lipids and fecal sterol excretion. Atherosclerosis, 36, 81-88.

35) Miettinen, A. P., and McNamara, D. J. (1981): Origins of fecal neutral steroids in rats. J. Lipid Res., 22, 485-495.

36) Quarfordt, S. H., and Goodman, D. S. (1967): Metabolism of doubly-labelled chylomicron cholesterylesters in the rat. J. Lipid Res., 8, 264-273. 